\title{
Evaluación del desempeño del conglomerado floricultor mejicano: Caso Baja California.
}

\section{Evaluation of the performance of the mexican floricultor cluster: Case Baja California}

\author{
Blanca Estela Bernal Escoto - Malena Portal Boza \\ Duniesky Feitó Madrigal
}

\section{MÉJICO}

Resumen: El presente artículo se enfoca en el análisis de resultados obtenidos al evaluar el grado de conformación del conglomerado floricultor del Estado de Baja California. La metodología utilizada es cuantitativa, de orden transaccional y de alcance descriptivo-aglomerativo. Para la recolección de la información se diseñó y aplicó un cuestionario previamente estructurado, dirigido a los 180 sujetos de estudio que conforman sus grupos de interés. El instrumento de recolección de datos se integra por 22 ítems, cuyos datos recabados fueron analizados con el paquete estadístico SPSS y el programa PyCX en Python, obteniéndose información relacionada con su estructura, tamaño, densidad, topología y propiedades estadísticas, así como el grado de interdependencia entre sus grupos de interés, permitiendo identificar al nodo hub.

Palabras clave: conglomerado empresarial; administración de la producción; evaluación de programas; administración de la cadena de suministro.

\begin{abstract}
The present article focuses on the analysis of the results obtained when evaluating the degree of conformation of the Floriculture Cluster of the state of Baja California. The methodology used is quantitative of transactional order and descriptive- Agglomeration. For the collection of information, a previously structured questionnaire was designed and applied to the 180 study subjects that make up their interest groups. The data collection instrument is composed of 22 items, whose data were analyzed with the SPSS statistical package and the PyCX program in Python, obtaining information related to its structure, size, density, topology and statistical properties, as well as the degree of interdependence Among its stakeholders, allowing the identification of the hub node.
\end{abstract}

Keywords: business conglomerate; production management; program evaluation; supply chain management.

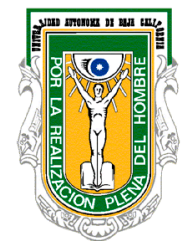

Blanca Estela Bernal es Licenciada en Administración de Empresas, Magíster en Administración de Empresas y Doctora en Planeación Estratégica para la Mejora del Desempeño. Es coordinadora del área de Mercadotecnia y profesora investigadora de tiempo completo en la Facultad de Contaduría y Administración, perfil PRODEP, de la Universidad Autónoma de Baja California, donde es miembro del cuerpo académico "Empresarialidad Social y Microfinanzas".

Contacto: blancab@uabc.edu.mx 


\title{
Avaliação do desempenho do grupo de floricultores mexicanos: Caso Baja Califórnia
}

Resumo: Este artigo centra-se na análise dos resultados obtidos, avaliando o grau de formação de grupo Floricultor de Baja California. A metodologia é escopo transacional quantitativo e descritivo-fim agglomerative. Para recolha de dados que foi concebido e implementado um questionário estruturado anteriormente, dirigido para os sujeitos do estudo 180 que compreendem os intervenientes; a recolha de dados do instrumento é composto de 22 itens, os dados obtidos foram analisados com o programa SPSS e PyCX Python obtenção de informações relacionadas com a sua estrutura, tamanho, densidade, topologia e propriedades estatísticas e o nível de interdependência entre as partes interessadas por forma a identificar o centro de nó.

Palavras-chave: conglomerado empresarial; gestão de produção; avaliação de programas; gestão da cadeia de suprimentos

\section{Introducción}

\begin{abstract}
A nivel mundial la comercialización de flores registró al cierre 1 de 2016 la cantidad de 1,381,791 toneladas con un valor de LUS\$7,9009,345.00 (Trade map, 2016), mientras que en Méjico los registros más recientes del valor de la producción de flores es de más de 6,408,005.48 toneladas con un valor de 296,079.88 millones de pesos mejicanos a 2014, siendo los Estados Unidos y Canadá los principales compradores de este tipo de productos; además, esta actividad económica es considerada como una fuente generadora de múltiples empleos (Servicio de Información Agropecuaria y Pesquera [SIAP], 2013). Las cifras anteriores proporcionan un panorama general de la importancia de estudio de este sector.
\end{abstract}

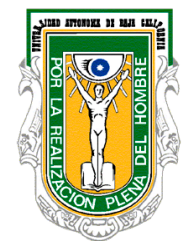

\footnotetext{
Malena Portal es Licenciada en Contabilidad y Finanzas, Magíster en Dirección de Empresas y Doctora en Economía. Es coordinadora del área de Finanzas y profesora investigadora de tiempo completo en la Facultad de Contaduría y Administración, perfil PRODEP, de la Universidad Autónoma de Baja California, donde es miembro del cuerpo académico “Empresarialidad Social y Microfinanzas”.

Contacto: mportal@uabc.edu.mx
}

Duniesky Feitó Madrigal es Ingeniero Industrial, Magíster en Administración de Negocios y Doctor en Economía. Es coordinador de la carrera en Administración y profesor investigador de tiempo completo en la Facultad de Contaduría y Administración, perfil PRODEP, de la Universidad Autónoma de Baja California, donde es miembro del cuerpo académico "Empresarialidad Social y Microfinanzas”.

Contacto duniesky.feito@uabc.edu.mx 
Dentro de los nuevos modelos de desarrollo, los conglomerados son los más utilizados por las micro, pequeñas y medianas empresas a nivel internacional como estrategia competitiva que les permite unir fuerzas contra los líderes del sector, los cuales suelen ser empresas grandes. Cabe destacar que un conglomerado es definido como "un grupo geográficamente denso de empresas e instituciones conexas, pertenecientes a un campo concreto, unidas por rasgos comunes y complementarias entre sí" (Porter, 1990: 36).

Con base en lo anterior, el pertenecer a un conglomerado o participar en una cadena de valor permite promover el aprendizaje y el mejoramiento sostenible de sus integrantes, (Padilla, Reyes, y Pérez, 2012: 57). Según esto, Porter (2005: 36) y Mitnik (2016: 261-263) establecen que las relaciones entre empresas derivadas del aprovechamiento colectivo permiten hacer un mejor aprovechamiento de las oportunidades del sector y promueven la confianza mutua mediante el valor compartido, el trabajo colaborativo, la innovación conjunta y el riesgo compartido, lo que implica un claro compromiso entre sus miembros.

Es por ello que el presente artículo tiene como propósito analizar y medir el desempeño de los grupos de interés que conforman el conglomerado floricultor del estado de Baja California, el cual es liderado por la empresa Asval Flowers Co. Para medir el grado de interdependencia y desempeño se utilizó la metodología de Sayama (2015: 4-5), quien propone un modelo de análisis de redes y sistemas complejos, el cual se basa en los trabajos de Python ${ }^{1}$ y su interfaz gráfica (Padilla, et al., 2012: 57), en donde las representaciones matemáticas se realizan a través de grafos, los cuales, a su vez, muestran las interacciones entre los elementos participantes mediante vértices -las líneas que unen a un nodo y otro- mostrando la distancia y peso existente entre cada uno.

\section{Revisión de literatura}

El concepto de conglomerado, también conocido como grupo, cúmulo o aglomeración, se originó en el siglo XIX, en Inglaterra, por Marshall (1890; citado por Serret, 2011), quien destacó la importancia de localizar a las organizaciones por su giro industrial, basándose en empresas inglesas y las dimensiones de su localización, específicamente de su concentración geográfica, caracterizándose por ser un grupo de industrias conectadas por importantes flujos de bienes y servicios. Inclusive, en su aportación inicial a este tema, Porter (citado por Serret, 2011: 20) define un grupo

1 Es una herramienta para desarrollar modelos y sistemas complejos (https:// www.python.org/). 
industrial como "un conjunto de industrias relacionadas a través de relaciones comprador-proveedor, o por tecnologías comunes, los compradores comunes o los canales de distribución, y los lugares comunes de trabajo".

De conformidad con Andersson, Schwaag, Sörvik y Wise, (2004: 29-31), los elementos que conforman un conglomerado derivan del incremento de la producción del sector, principalmente lo que requiere de un enfoque integrador, que presupone incluir las dimensiones social, política y cultural (Véase tabla 1).

Tabla 1. Elementos clave para la creación de un conglomerado

\begin{tabular}{|c|l|}
\hline $\begin{array}{c}\text { Concentración } \\
\text { geográfica }\end{array}$ & $\begin{array}{l}\text { Elemento esencial en el surgimiento de los clústeres, } \\
\text { considera los aspectos que sustentarán la importancia } \\
\text { de la cercanía geográfica. } \\
\text { denominadorión o común } \\
\text { Por lo general, los actores del clúster se relacionan } \\
\text { alrededor de una actividad central. } \\
\text { Se agrupan básicamente en cuatro categorías: } \\
\text { empresas, gobiernos, comunidad científica, } \\
\text { instituciones financieras. } \\
\text { Las conexiones e interrelaciones entre los actores } \\
\text { pueden tener características tanto de competencias } \\
\text { como de colaboración. } \\
\text { encadenamiento }\end{array}$ \\
Masa crítica & $\begin{array}{l}\text { Es un concepto relacionado con las economías } \\
\text { de escala y de alcance, así como con el patrón de } \\
\text { dependencia. } \\
\text { El clúster es un modo de organización de largo plazo } \\
\text { y, por lo tanto, tiene un ciclo de vida que comprende } \\
\text { varias etapas. } \\
\text { Se asocia al conocimiento que se genera por la } \\
\text { interacción social. Es el proceso mediante el cual la } \\
\text { firma domina y pone en práctica diseños de productos } \\
\text { y procesos de manufactura que son nuevos para ella. }\end{array}$ \\
Innovación &
\end{tabular}

Fuente: Adaptado de "The Clúster Policies Whitebook," por Andersson,

Schwaag, Sörvik, y Wise, (2004: 29-31).

\section{Metodología}

Se trabajó con una metodología cuantitativa de corte transaccional, con alcance descriptivo-aglomerativo, centrada en describir las condiciones del área de estudio, las principales características de los sujetos participantes, así como el cálculo del tamaño de la muestra requerida; se describieron los materiales que se utilizaron para su elaboración y, por último, se enlistaron los pasos que conforman el procedimiento general 
que se siguió para la obtención de los datos para evaluar el desempeño de conglomerado estudiado.

\section{Medios materiales}

Los materiales utilizados fueron de dos tipos: 1) las fuentes secundarias, entendiéndose estas como todo documento, reporte, artículo de apoyo utilizado ya existente y que fueron consultados y agregados en las referencias bibliográficas; 2) fuentes primarias, se realizaron entrevistas programadas con los sujetos de estudio y se aplicó un cuestionario que consta de 22 ítems, con preguntas cerradas, dicotómicas, opción múltiple y de valoración; dichos ítems fueron diseñados para el cumplimiento del objetivo.

Posteriormente, el instrumento se sometió a juicio de 10 especialistas en la temática con la finalidad de medir la pertinencia de los ítems, en donde, mediante una cédula de evaluación, se valoró cada pregunta como "adecuada" o "no adecuada", obteniéndose como resultado un índice Kappa de 0.80 , lo que significa que, de conformidad con los jueces, la fiabilidad de los reactivos es considerable o sustancial (Cerda \& Villalobos, 2008, 57).

Para el análisis de datos se utilizó el paquete estadístico SPSS versión 20, lo que permitió identificar el porcentaje de interrelación entre los participantes (nodos) de la cadena productiva; también facilitaron la medición del grado de interrelación entre los productores locales de flor (UP), los proveedores de la cadena ( $p$ ) y los clientes (C) a los que el conglomerado dirige sus esfuerzos, evaluando, a su vez, de conformidad con la fuerza que cada participante ejerce, sus principales características tales como el tamaño, la densidad, la topología y propiedades estadísticas (Sayama, 2015: 4-5); para ello, se hizo uso del programa PyCX.

\section{Descripción del área de estudio}

Para comprender las condiciones en que se desarrolló la investigación, es primordial destacar que la recogida de la información se llevó a cabo en tres de los cinco municipios del estado de Baja California (Tijuana, Playas de Rosarito y Ensenada), en donde se localizan 10 de las 16 unidades productoras pertenecientes al sector florícola, situadas en las localidades de La Misión, Rancho los Amigos, Familia Reséndiz, Estación Coahuila (Km. 57), Colonia Ahumada, Rancho los Alisos, El Descanso, el Roble, Emiliano Zapata, Tijuana, Rancho la Avena, el Ejido Lázaro Cárdenas y el Rancho el Toro (INEGI, 2015). Con respecto a los clientes, estos se clasifican en nacionales y extranjeros, ubicados en Tijuana y Estados Unidos, principalmente en Carlsbad, San Ysidro y San Diego, California; de 
igual forma, los proveedores se localizan mayormente en Baja California, Estados Unidos y Holanda (Valenzuela, J. \& Aceves, Y., 2016:15).

\section{Sujetos de estudio}

Con relación al tipo de sujetos de estudio objeto de esta investigación, es preciso mencionar que pertenecen al mercado de industria -i.e., empresas que venden a otras empresas-; dichos sujetos integran el conglomerado y son comúnmente conocidos como grupos de interés. Con lo anterior en mente, se identificó una población total de 180 participantes, los cuales fueron tomados en su totalidad como sujetos de estudio, quienes, a su vez, fueron clasificados de acuerdo con las características mencionadas en la siguiente Tabla 2, así como el número y porcentaje que cada uno de estos representan en el conglomerado.

Tabla 2. Características de los sujetos de estudio.

\begin{tabular}{|c|c|c|c|}
\hline Grupos de interés & Características & $\mathbf{N}$ & $\%$ \\
\hline $\begin{array}{l}\text { Productor sector } \\
\text { primario }\end{array}$ & $\begin{array}{l}\text { Especialistas en la producción, } \\
\text { comercialización y logística } \\
\text { de agronegocios (flor de corte y } \\
\text { agroproductos). } \\
\text { Cuentan con un promedio de entre } 1 \text { y } 5 \\
\text { empleados. } \\
\text { Presentan elevada rotación de personal. } \\
\text { El tipo de producción predominante es } \\
\text { en invernadero. } \\
\text { Se caracterizan por trabajar bajo la } \\
\text { informalidad. }\end{array}$ & 15 & 8 \\
\hline \multirow[t]{2}{*}{$\begin{array}{l}\text { Proveedores de } \\
\text { insumos }\end{array}$} & $\begin{array}{l}\text { Nacionales (Baja California: semillas, } \\
\text { esquejes, fertilizantes y maquinaria). }\end{array}$ & 26 & 14 \\
\hline & $\begin{array}{l}\text { Extranjeros, provenientes de Holanda } \\
\text { (material genético, como bulbos y } \\
\text { semillas); y de Estados Unidos (material } \\
\text { genético, esquejes y semillas, y material } \\
\text { para invernadero, como madera y acero). }\end{array}$ & 2 & 1 \\
\hline $\begin{array}{l}\text { Proveedores de } \\
\text { servicios }\end{array}$ & $\begin{array}{l}\text { Ofrecen servicios de logística, centros de } \\
\text { acopio y transporte. }\end{array}$ & 7 & 4 \\
\hline \multirow[b]{2}{*}{$\begin{array}{l}\text { Clientes comerciales } \\
\text { mayoristas }\end{array}$} & Nacionales localizados en Baja California. & 2 & 1 \\
\hline & $\begin{array}{l}\text { Extranjeros localizados en Estados } \\
\text { Unidos (Carlsbad, San Ysidro y San } \\
\text { Diego, California). } \\
\text { Ventas anuales de US\$1.6 millones }\end{array}$ & 8 & 4 \\
\hline
\end{tabular}




\begin{tabular}{|c|c|c|c|}
\hline Grupos de interés & Características & $\mathbf{N}$ & $\%$ \\
\hline Distribuidores & $\begin{array}{l}\text { Extranjeros: intermediación del } \\
\text { producto hacia el cliente minorista, } \\
\text { lo hacen llegar a más de } 500 \text { florerías } \\
\text { ubicadas en el sur de California. } \\
\text { Ventas anuales de US\$7.5 millones }\end{array}$ & 5 & 3 \\
\hline $\begin{array}{l}\text { Organismos } \\
\text { gubernamentales y } \\
\text { entidades civiles }\end{array}$ & $\begin{array}{l}\text { Consejo Agrícola Mejicano: } \\
\text { SAGARPA, UABC, CDT y el Consejo } \\
\text { Mejicano de la Flor. }\end{array}$ & 10 & 6 \\
\hline $\begin{array}{l}\text { Comunidad en } \\
\text { general }\end{array}$ & $\begin{array}{l}\text { Genera } 600 \text { empleos al año, diversidad } \\
\text { de género (jornaleros). } \\
\text { Escolaridad básica. }\end{array}$ & 70 & 39 \\
\hline \multirow[t]{2}{*}{$\begin{array}{l}\text { Socios } \\
\text { propietarios }\end{array}$} & $\begin{array}{l}\text { En su mayoría con educación media. } \\
\text { Formación empírica. }\end{array}$ & 35 & 19 \\
\hline & Total & 180 & 100 \\
\hline
\end{tabular}

Fuente: Elaboración propia con base en el análisis de los datos.

SAGARPA: Secretaría de Agricultura, Ganadería, Desarrollo Rural, Pesca y Alimentaria (2015)

UABC: Universidad Autónoma de Baja California.

CDT: Consejo de Desarrollo Económico de Tijuana.

\section{Planteamiento del problema}

Se desconocía el grado de contribución entre los participantes (nodos) de la cadena productiva hacia dentro del conglomerado y no existía un método que permitiera medir la interrelación entre los integrantes de los grupos de interés del conglomerado; en lo sucesivo serán identificados de conformidad con la siguiente Tabla 3 Simbología de los participantes, evaluando, a su vez, de conformidad con la fuerza que cada participante ejerce, sus principales características: tamaño, densidad, topología y propiedades estadísticas. Para ello, se hizo uso del programa PyCX. (Sayama, 2015: 4-5).

Tabla 3. Simbología de los participantes

\begin{tabular}{|l|l|}
\hline \multicolumn{1}{|c|}{ Simbología } & \multicolumn{1}{|c|}{ Participante } \\
\hline UP & Unidades productivas \\
P & Proveedores \\
C & Clientes \\
\hline
\end{tabular}

Fuente: Elaboración propia con base en (Sayama, 2015: 4-5) 
Para logar el objetivo de estudio se implementó el método de sistemas complejos. Primeramente, se requiere puntualizar que todo conglomerado es tipificado a través de redes o grafos, lo cual se define como: un conjunto de objetos llamados nodos o vértices que están conectados juntos, cuyas conexiones entre los nodos se denominan bordes o enlaces (Geyer y Cairney, 2015: 6).

Por otra parte, las redes pueden ser analizadas por sus características, siendo la más representativa su estructura, la cual comprende el análisis de su tamaño, la densidad, la topología y propiedades estadísticas; este análisis será posible mediante el uso del programa PyCX, el cual es un marco para la simulación de sistemas complejos basado en Python y su interfaz gráfica, la que permite a los usuarios realizar simulaciones dinámicas en tiempo real (Wong, 1989; citado por Sayama, 2015, 5).

Las propiedades estructurales más básicas de la red son su tamaño y la densidad, debido a que estas determinan la cantidad de elementos que la conforman; por su parte, el tamaño de una red se caracteriza por el número de nodos y bordes en ella, mientras que la densidad de esta indica el nivel de interconexión entre sus nodos, en donde, entre mayor densidad mayor número de nodos interconectados. Para redes direccionadas se utiliza la siguiente fórmula: $P(G)=m /(n(n-1))$, en donde " $m$ " representa el número de conexiones y " $n$ " el número de nodos (Sayama, 2015: 7).

Además, para evaluar el conglomerado floricultor estudiado se determinó el índice de agrupamiento que, de conformidad con Sayama (2015), representa el número de aristas o enlaces $(\mathrm{m})$ conectados a un nodo, identificando a cada uno de sus integrantes y el nivel de interdependencia entre estos; de igual forma, se diseñó la lista de adyacencia cuyo propósito es representar a cada nodo con respecto al número de nodos vecinos. Dicha lista sirvió para representar la fuerza de cada nodo con relación al nodo concentrador o "hub" -el que emplea o tiene algún tipo de relación comercial con el resto de los participantes- i.e., un concentrador es un nodo con un alto grado de conexiones con otros nodos (Geyer y Cairney, 2015: 6).

Finalmente, se generó la matriz de adyacencia, la cual para una red de " $\mathrm{N}$ " nodos es considerada como una matriz de unos y ceros, donde un uno indica la presencia del borde correspondiente en la red; si los bordes en una red están dirigidos, es decir, apuntando en una sola dirección, la red se denomina red dirigida; cuando se dibuja una red dirigida, los bordes se dibujan típicamente como flechas que indican la dirección (Sayama, 2015). 
Figura 1. Pasos para tipificar los conglomerados.

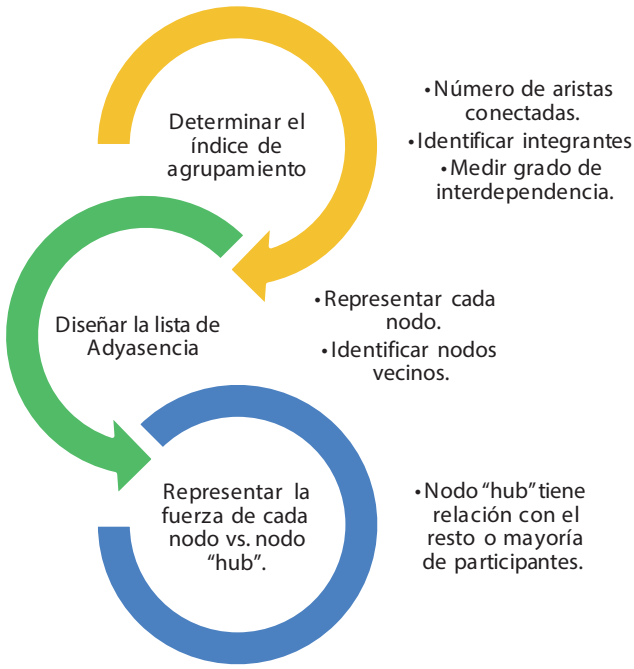

Fuente: Elaboración propia con base en Sayama (2015: 7).

\section{Resultados}

El diagnóstico de las cadenas productivas es de gran utilidad para evaluar el desempeño de sus integrantes, tales como: la eficiencia, calidad de productos, sostenibilidad y equidad. En este apartado del instrumento se identificó el porcentaje de relación comercial que cada productor guarda con el resto, así como con los intermediarios mayoristas y proveedores.

Inicialmente, se identificó la localización física de cada uno de los grupos de interés a fin de determinar la proximidad geográfica y cultural de estos, ofreciendo a los participantes información sobre sus vecinos comerciales, lo que propició relaciones más estrechas y otras ventajas difíciles de alcanzar por competidores alejados de la red. Los resultados arrojaron que los municipios que alojan mayormente a los participantes son Playas de Rosarito, Tijuana y Ensenada (Véase figura 2).

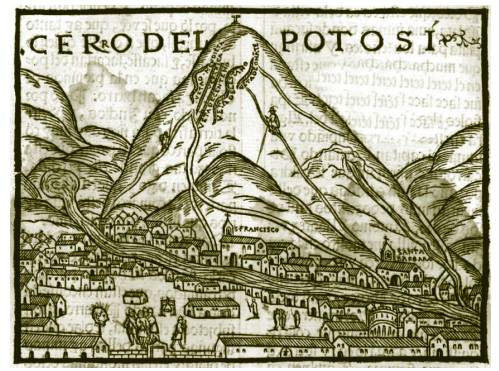


Figura 2. Ubicación de los grupos de interés.

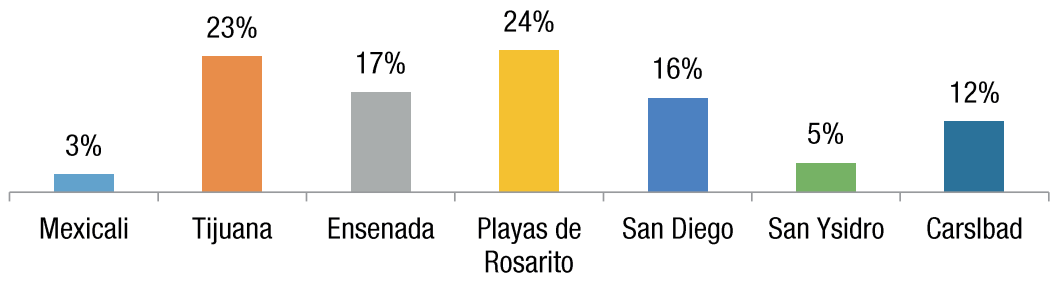

Fuente: Elaboración propia con base en la recopilación de la información.

Con respecto al tamaño de las empresas participantes, se utilizó la clasificación propuesta por la Secretaría de Economía con respecto al personal ocupado, predominando las micro, pequeñas y las medianas empresas. (Véase figura 3).

Figura 3. Tamaño de las empresas participantes.
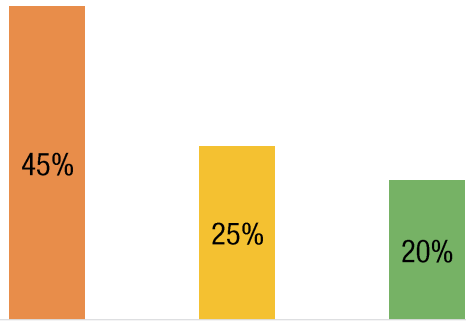

\section{$10 \%$}

Micro

Pequeña

Mediana

Grande

Fuente: Elaboración propia con base en la recopilación de la información.

Con relación al número de asociados estratégicos que cada participante posee, estos se muestran por tipo y número en la siguiente figura 4. Es importante hacer mención que el $91 \%$ afirmó contar con por lo menos un asociado, mismos que pertenecen al grupo de productores locales, seguido de los proveedores y transportistas.

Figura 4. Tipo y número de asociados estratégicos que posee cada participante

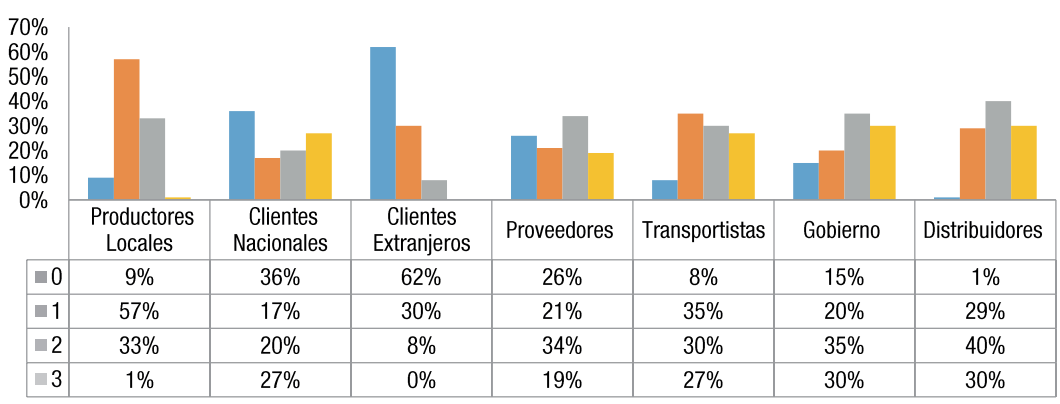

Fuente: Elaboración propia con base en la recopilación de la información. 
Para tal efecto, se les preguntó a los empresarios de las Mipyme productoras de flor de la región en qué porcentaje tenían relaciones comerciales con otros productores. (Para efectos de confidencialidad, los productores o unidades económicas serán representadas en lo sucesivo con letras, siendo la empresa $A$ la empresa líder). Estos respondieron que mayormente con el Productor A (73\%), seguido del productor $\mathrm{F}$ (60\%) y de los productores C y G (38\%) respectivamente (Véase figura 5). El comportamiento anterior es debido a que el productor $A$ es quien concentra la mayor transacción comercial con productores locales, clientes y proveedores locales y extranjeros.

Figura 5. Porcentaje de relación comercial entre productores florícolas de Baja California.

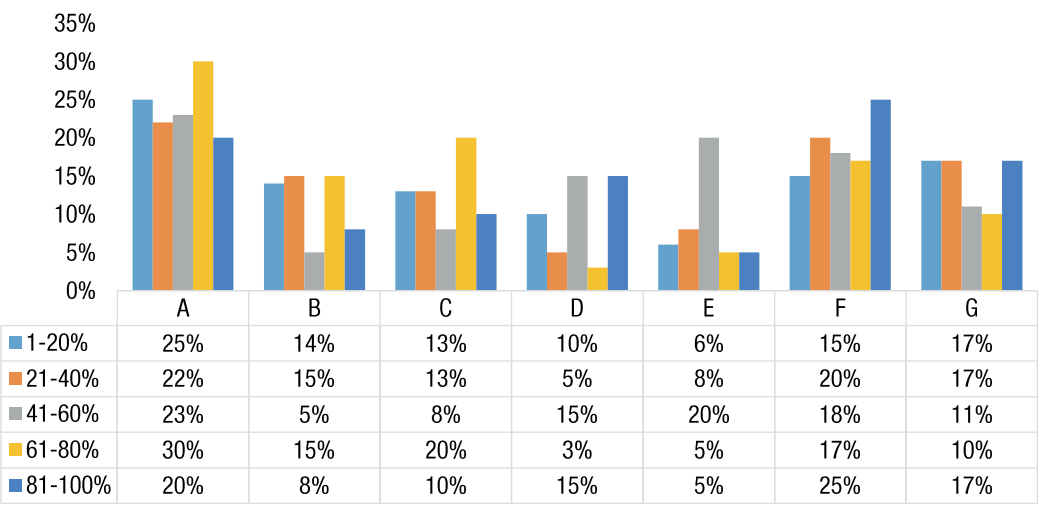

Fuente: Elaboración propia con base en la recopilación de la información.

* La empresa A representa a la empresa sujeta de estudio: Asval Flowers Co., líder del conglomerado.

Con la información anterior se pudo determinar el índice de agrupamiento que cada participante presenta, en donde el nodo (UP1), como ya se mencionó, representa a la empresa sujeta de estudio "Asval Flowers Co.", que, a su vez, resulta ser el nodo "hub" (concentrador), lo que implica que emplea o tiene relación comercial directa con el resto de los productores, mostrando el mayor índice de agrupamiento con respecto a los demás (0.60). De igual forma, se puede identificar la relación comercial de los demás con base en el índice que presentan -por ejemplo, el nodo dos (UP2) tiene relación directa con tres vecinos (UP3, UP4 y UP6) dándoles trabajo dentro del conglomerado, mientras que el nodo cinco (UP5) solamente provee al nodo "hub", pero en una mayor proporción que el resto- (Véase tabla 4). Lo anterior se interpreta de la siguiente manera: en el supuesto caso de que (UP5=E) -nodo cinco- decida terminar relaciones comerciales con el conglomerado, el desempeño del grupo se verá seriamente afectado debido a su representativa participación actual (0.87), lo que se traduce en la pérdida de un eslabón clave de la cadena 
productiva del conglomerado; mientras que al perder la colaboración del productor tres $(U P 3=C)$ dejaría sin empleo al productor $B$ y muchas familias de la localidad, lo que representaría un gran déficit comercial para la región.

Tabla 4. Lista de adyacencia de las unidades productivas

\begin{tabular}{|c|c|c|}
\hline Empleador & Productor & Producción (\%) \\
\hline UP1 & UP2 & 0.60 \\
UP1 & UP3 & 0.25 \\
UP1 & UP4 & 0.80 \\
UP1 & UP5 & 0.87 \\
UP1 & UP6 & 0.25 \\
UP1 & UP7 & 0.40 \\
UP2 & UP3 & 0.37 \\
UP2 & UP4 & 0.10 \\
UP4 & UP6 & 0.25 \\
UP4 & UP7 & 0.20 \\
\hline
\end{tabular}

Fuente: Elaboración propia con base en la recopilación de la información.

A partir de la lista de adyacencia anterior se obtuvo el peso de cada nodo, lo que representa el poder de negociación de los competidores, siendo UP1 el que obtuvo un mayor puntaje -por ejemplo, para calcular el peso del UP1 se sumó el porcentaje de producción que los demás productores le aportan, dando un peso de 3.17, lo que refuerza que este es considerado el nodo "hub", seguido por UP4, ya que este tiene relación con cuatro vecinos productores más-. Cabe destacar que los nodos más débiles son UP6 y UP7 (Véase tabla 5).

Tabla 5. Peso del nodo

\begin{tabular}{|c|c|}
\hline Nodo & Peso del nodo* \\
\hline UP1 & 3.17 \\
UP2 & 1.07 \\
UP3 & 0.62 \\
UP4 & 1.35 \\
UP5 & 0.87 \\
UP6 & 0.50 \\
UP7 & 0.60 \\
\hline
\end{tabular}

Fuente: Elaboración propia con base en la lista de adyacencia de las unidades productivas

* Donde UP1 $=(0.60+0.25+0.80+0.87+0.25+0.40)=3.17$ 
Una vez elaborada la lista de adyacencia y calculado el peso de cada nodo, es preciso elaborar la matriz de adyacencia de los productores locales, la que indica el grado de dependencia entre cada nodo o vértice que lo conforman, siendo Asval Flowers Co. (UP1) la empresa independiente, y UP5 la empresa que más depende de los demás (Véase tabla 6).

Tabla 6. Matriz de adyacencia del conglomerado de productores florícolas de Baja California

\begin{tabular}{|c|c|c|c|c|c|c|c|c|}
\hline \multirow{2}{*}{$\frac{7}{\circ}$} & \multicolumn{7}{c|}{ Productor } \\
\cline { 2 - 9 } z & $\begin{array}{c}\text { A } \\
\text { (Asval) }\end{array}$ & B & C & D & E & F & G & \\
\cline { 2 - 9 } & UP1 & UP2 & UP3 & UP4 & UP5 & UP6 & UP7 & Grado* \\
\hline UP1 & 0 & 1 & 1 & 1 & 1 & 1 & 1 & 6 \\
UP2 & 1 & 0 & 1 & 1 & 0 & 0 & 0 & 3 \\
UP3 & 1 & 1 & 0 & 0 & 0 & 0 & 0 & 2 \\
UP4 & 1 & 1 & 0 & 0 & 0 & 1 & 1 & 4 \\
UP5 & 1 & 0 & 0 & 0 & 0 & 0 & 0 & 1 \\
UP6 & 1 & 0 & 0 & 1 & 0 & 0 & 0 & 2 \\
UP7 & 1 & 0 & 0 & 1 & 0 & 0 & 0 & 2 \\
\hline
\end{tabular}

Fuente: Elaboración propia con base en la recopilación de la información.

Nota. ${ }^{*}$ Representa el número de empresas que dependen de cada participante.

Finalmente, la topología del conglomerado muestra los sentidos de interrelación entre sus miembros, representado por líneas más gruesas, y el grado de participación entre estos, simbolizado por el tamaño del nodo, ya que entre más lejos se encuentra un nodo, mayor es la dependencia con el nodo "hub". Esto se puede interpretar de la siguiente forma: los nodos (UP4) y (UP2) son los más cercanos al nodo "hub" (UP1) y el nodo (UP5) es el que depende directamente del nodo "hub" (UP1), ya que no tiene relaciones comerciales con nadie más; además, se puede apreciar que se trata de una red direccionada, ya que se muestran los sentidos de interdependencia entre cada uno (Véase figura 6).

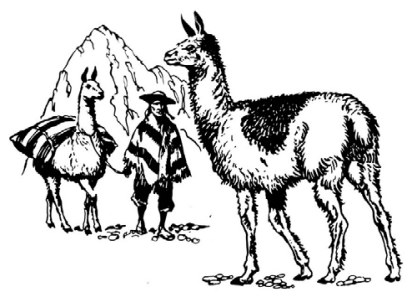


Figura 6. Topología del conglomerado productor.

* El peso se indica en las líneas de interconexión.

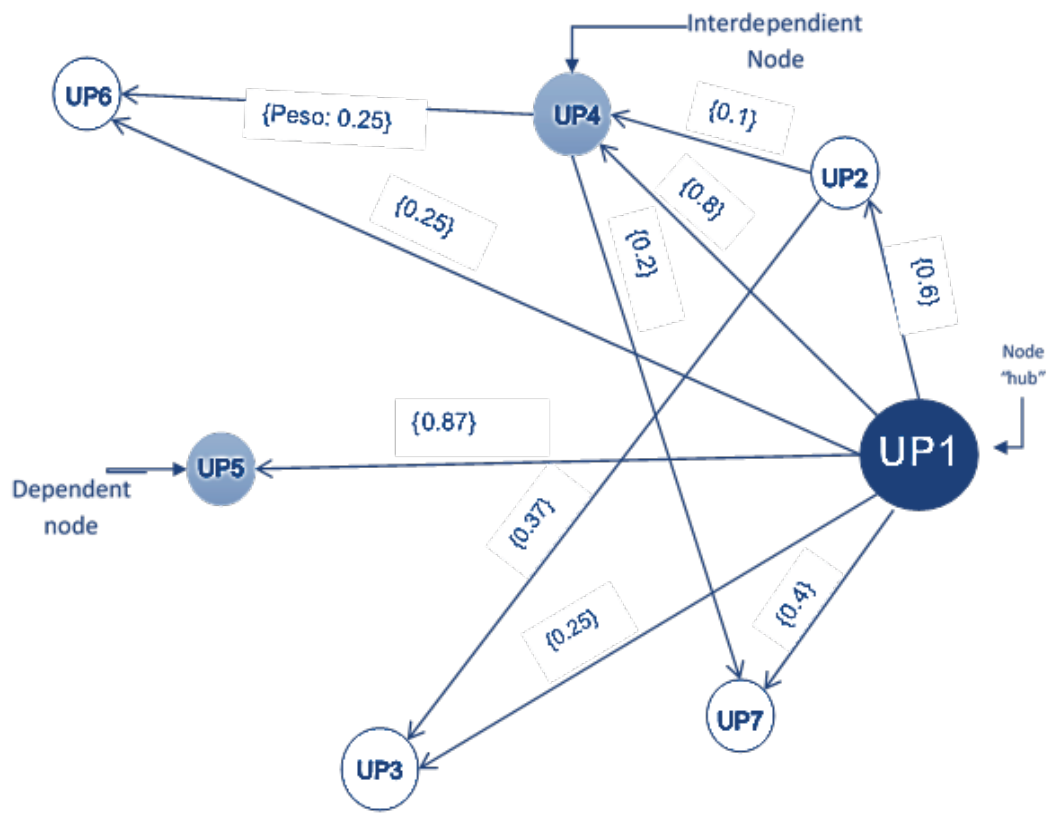

Fuente: Elaboración propia con base en la recopilación de información mediante PyCX basado en Python (www.python.org/).

Con respecto a la direccionalidad de la red, el grafo permite identificar quién es el empleador y quién es el que provee; esto es posible debido a que el extremo del conector del primero es más delgado en comparación con el que entra al nodo del segundo, el cual es más grueso; así mismo, en relación con la densidad de la red del conglomerado para la floricultura para Baja California se utilizó la siguiente fórmula: $P(G)=\frac{m}{n(n-1)}$

Se determinó que $\mathrm{m}$ representa el número de conexiones (10) y $\mathrm{n}$ el número de nodos (7), obteniéndose una densidad de 0.23 , lo que indica que la red posee una amplia interrelación entre sus partes, demostrando que existe una integración horizontal entre los competidores del sector florícola. El procedimiento se muestra a continuación:

$$
P(G)=\frac{10}{7(7-1)}=0.23
$$

Uno de los beneficios de dibujar el comportamiento de los participantes es que permite representar visualmente el dinamismo cambiante que presenta. Mediante la matriz de adyacencia se logra conocer el número 
de aristas conectadas a un nodo, lo que representa el dinamismo y el sentido de la interdependencia.

El segundo paso del análisis de la cadena productiva consiste en identificar a los clientes con los que el conglomerado realiza sus tratos comerciales, identificándose a cinco de origen extranjero (sur de Estados Unidos), en donde únicamente Asval Flowers Co. tiene trato directo con ellos, seguido de los intermediarios " $\mathrm{B}$ " $\mathrm{y}$ " $\mathrm{C}$ "; los porcentajes en los cuales se interrelacionan se presentan en la siguiente figura 7. Es importante mencionar que el $100 \%$ de los productores locales tienen relación directa con el intermediario $\mathrm{A}$.

Figura 7. Porcentaje de participación con intermediarios mayoristas.

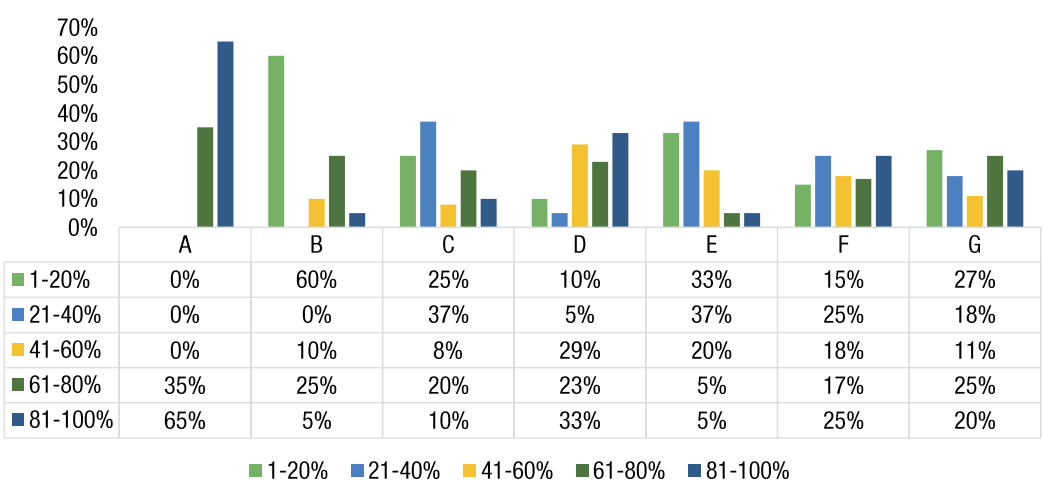

Fuente: Elaboración propia con base en la recopilación de la información.

De igual forma, se determinó la lista de adyacencia para los cinco clientes mayoristas con los que las empresas tienen relaciones comerciales a la fecha (ver tabla 7). Como se puede observar, el cliente uno (C1) es considerado como su cuenta clave, debido a que le compra el $35 \%$ de su producción anual. Con fundamento en lo anterior, no fue necesario realizar la matriz de adyacencia, ya que el grado de participación es igual a uno en todos los casos.

Tabla 7. Lista de adyacencia entre producto concentrador y clientes

\begin{tabular}{|c|c|c|}
\hline Productor & Cliente & Compra anual (\%) \\
\hline UP1 & C1 & 0.35 \\
UP1 & C2 & 0.10 \\
UP1 & C3 & 0.15 \\
UP1 & C4 & 0.25 \\
UP1 & C5 & 0.15 \\
\hline
\end{tabular}

Fuente: Elaboración propia con base en la recopilación de la información. 
Para determinar la densidad de la red con respecto a sus clientes, se identificó que $\mathrm{m}$ representa el número de conexiones (5) y $\mathrm{n}$ el número de nodos (5), obteniéndose una densidad de 0.50 , lo que indica que la red posee una amplia interrelación entre sus clientes comerciales y el nodo "hub" (Asval Flowers Co). El procedimiento se muestra a continuación:

$$
P(G)=\frac{5}{5(5-1)}=0.50
$$

Figura 8. Topología del conglomerado productor respecto a los clientes mayoristas. *El peso se indica en las líneas de interconexión.

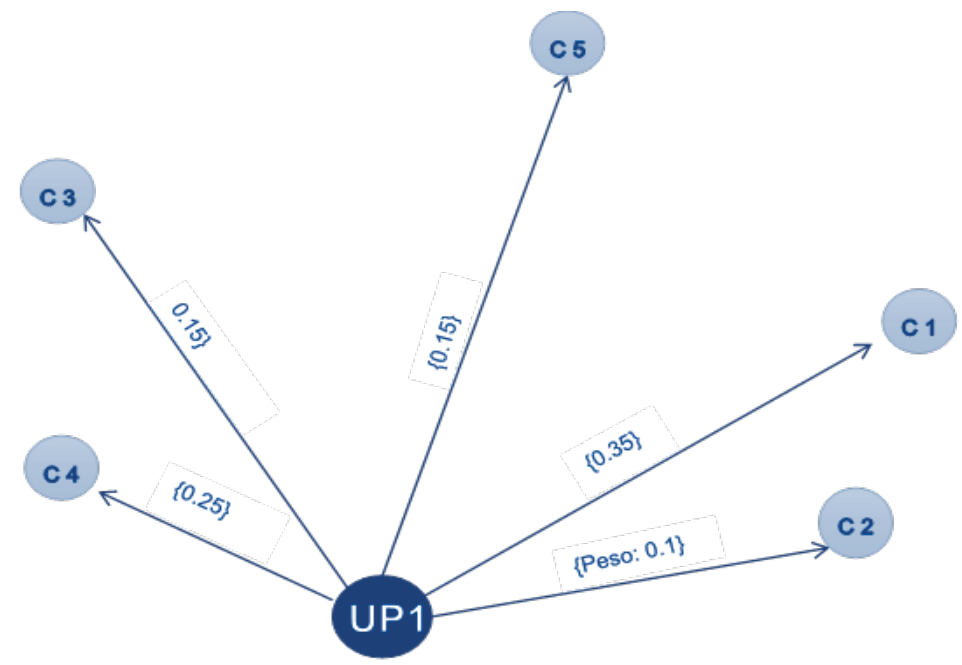

Fuente: Elaboración propia con base en recopilación de información, mediante programa PyCX basado en Python.

El tercer paso del diagnóstico de las cadenas productivas consiste en identificar si las empresas florícolas que integran el conglomerado están afiliadas a alguna asociación, esto con la finalidad de conocer los motivos que favorecen la participación colaborativa. Cabe señalar que los participantes afirmaron que la intervención de las sociedades civiles y gubernamentales, los sindicatos y los grupos de protección del medio ambiente podrían ser de gran influencia en la promoción de negocios de producción de flores de Baja California, aumentando la presión de sus grupos de interés con respecto a la implementación y desarrollo de avances tecnológicos en el sector.

Con respecto al principal organismo al cual están afiliados, el 70\% de los empresarios floricultores afirmaron asociarse a Sagarpa (2015) con el objetivo de obtener la certificación referente a las normas y recomendaciones técnicas aplicables a la producción, procesamiento y 
transporte de alimentos, orientados a cuidar la salud humana, proteger el medio ambiente y mejorar las condiciones de los trabajadores y su familia, así como la obtención de créditos financieros mediante el programa de apoyo directo al campo (Pro Agro, 2015).

Asimismo, el $40 \%$ de los productores afirmó pertenecer principalmente al Consejo Agrícola de Baja California, que busca, a través del Consejo Nacional Agropecuario, defender la libre empresa en el campo, promover el desarrollo sostenible del sector agroalimentario, propiciar el ordenamiento de las cadenas agroalimentarias, representar al sector agropecuario privado del país, propiciar el ordenamiento de sistemas de comercialización modernos e integrados y promover la capacitación de los productores, entre otros.

De igual forma, el $25 \%$ de los floricultores mencionó acudir al Consejo Mejicano de la Flor A.C, ya que este actúa como representante y gestor de los intereses del sector florícola nacional ante los tres niveles de gobierno: federal, estatal y municipal, así como ante otros organismos e instituciones públicas y privadas, nacionales y del extranjero, en apoyo a la solución de problemas y demandas justas de los productores.

Finalmente, la Universidad Autónoma de Baja California y Wageningen University de Holanda fungen como encargadas de desarrollar investigaciones que permitan a los productores obtener una mayor eficacia en sus procesos de producción y al resto de la cadena incrementar su competitividad.

En otro orden de ideas, el primer paso para el análisis de la cadena productiva de los proveedores consiste en identificar a los proveedores que participan en la cadena productiva de este sector económico, en donde se identificó que a nivel estatal, de conformidad con el directorio estadístico nacional de unidades económicas (DENUE) de INEGI (2015), la conforman 233 empresas, las cuales proveen de estos insumos a los participantes de la cadena productiva del sector florícola de Baja California, a su vez, con dos proveedores del extranjero (Holanda y Canadá).

Posteriormente, la flor cortada es transportada y trasladada a cuartos o almacenes, conformada por cinco unidades económicas, mientras que en la fase de empaque se necesitan plástico y cartón, en donde el sector proveedor de estos materiales es crítico porque afectan la calidad durante el trasporte; al final están las comercializadoras, quienes concentran el producto principalmente en Carlsbad y lo distribuyen a lo largo del sur de California en estado de bouquet.

A partir de la encuesta, se detectaron a los 16 proveedores de la región que actualmente participan de forma significativa con por lo menos uno de los grupos de interés, identificándose al proveedor cinco (p5) 
como aquel que posee mayor grado de proveeduría, seguido por el proveedor ocho (p8) y quince (p15), siendo Asval Flowers Co (UP1) el común denominador de todos ellos, considerándose al nodo "hub" del aglomeramiento. Con dicha información, se diseñó la lista de adyacencia entre los productores locales (UP) y los proveedores del sector con los que tienen relación, así como el porcentaje de insumos que aporta cada uno a la cadena productiva, siendo los proveedores ( $p 5$, p8 y p15) los que contribuyen mayoritariamente con los insumos necesarios para que su cadena de valor sea eficiente y competitiva (Véase tabla 8).

Tabla 8. Lista de adyacencia

\begin{tabular}{|c|c|c|c|c|c|c|c|c|}
\hline 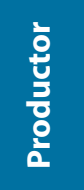 & 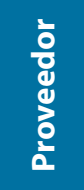 & 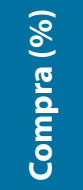 & 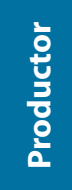 & 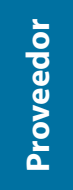 & $\frac{\overparen{\varrho}}{\frac{0}{0}}$ & $\begin{array}{l}\text { ț } \\
\text { ț } \\
\text { ơ }\end{array}$ & $\begin{array}{l}\text { 흥 } \\
\text { ơ } \\
\text { o }\end{array}$ & 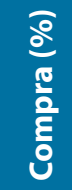 \\
\hline UP1 & p2 & 0.17 & UP3 & p6 & 0.11 & UP5 & p14 & 0.1 \\
\hline UP1 & p3 & 0.22 & UP3 & p7 & 0.26 & UP6 & $\mathrm{p} 1$ & 0.1 \\
\hline UP1 & p5 & 0.36 & UP3 & p8 & 0.1 & UP6 & p3 & 0.14 \\
\hline UP1 & p6 & 0.25 & UP3 & p9 & 0.12 & UP6 & p4 & 0.12 \\
\hline UP1 & p7 & 0.2 & UP3 & $\mathrm{p} 12$ & 0.21 & UP6 & p6 & 0.1 \\
\hline UP1 & p8 & 0.3 & UP4 & $\mathrm{p} 1$ & 0.12 & UP6 & $\mathrm{p} 12$ & 0.11 \\
\hline UP1 & p9 & 0.17 & UP4 & p2 & 0.13 & UP7 & $\mathrm{p} 1$ & 0.07 \\
\hline UP1 & $\mathrm{p} 12$ & 0.15 & UP4 & p6 & 0.11 & UP7 & p9 & 0.1 \\
\hline UP1 & p14 & 0.25 & UP4 & p7 & 0.11 & & & \\
\hline UP1 & $\mathrm{p} 15$ & 0.35 & UP4 & p8 & 0.1 & & & \\
\hline UP1 & p16 & 0.25 & UP4 & p12 & 0.12 & & & \\
\hline UP2 & p4 & 0.25 & UP4 & $\mathrm{p} 13$ & 0.15 & & & \\
\hline UP2 & p7 & 0.11 & UP5 & p7 & 0.05 & & & \\
\hline UP2 & p10 & 0.12 & UP5 & p11 & 0.15 & & & \\
\hline UP2 & $\mathrm{p} 12$ & 0.1 & UP5 & $\mathrm{p} 12$ & 0.1 & & & \\
\hline UP3 & p2 & 0.07 & UP5 & p13 & 0.1 & & & \\
\hline
\end{tabular}

Fuente: Elaboración propia con base en la recopilación de la información.

Partiendo de la lista anterior se determinó el peso de cada nodo, el cual representa el poder de negociación de los proveedores dentro de la cadena productiva, resultando ser el proveedor 12 (p12) el de mayor peso ya que suministra a todos los productores que integran el conglomerado a excepción del productor siete (UP7), seguido de los proveedores siete (p7) y seis (p6), los cuales tienen relaciones comerciales con cuatro de 
los productores locales respectivamente, resultando ser el proveedor 11 (P11) el de menor peso (Véase tabla 9).

Tabla 9. Peso del nodo

\begin{tabular}{|c|c|c|c|}
\hline Nodo & Peso & Nodo & Peso \\
\hline $\mathrm{p} 1$ & 0.29 & $\mathrm{p} 9$ & 0.39 \\
$\mathrm{p} 2$ & 0.37 & $\mathrm{p} 10$ & 0.29 \\
$\mathrm{p} 3$ & 0.36 & $\mathrm{p} 11$ & 0.22 \\
$\mathrm{p} 4$ & 0.37 & $\mathrm{p} 12$ & 0.79 \\
$\mathrm{p} 5$ & 0.36 & $\mathrm{p} 13$ & 0.35 \\
$\mathrm{p} 6$ & 0.57 & $\mathrm{p} 14$ & 0.35 \\
$\mathrm{p} 7$ & 0.73 & $\mathrm{p} 15$ & 0.35 \\
$\mathrm{p} 8$ & 0.50 & $\mathrm{p} 16$ & 0.25 \\
\hline
\end{tabular}

Fuente: Elaboración propia con base en la recopilación de la información.

Posteriormente se diseñó la matriz de adyacencia entre los proveedores participantes en la cadena productiva y los productores locales, a fin de determinar el grado de interdependencia entre estos (Véase tabla 10).

Tabla 10. Matriz de adyacencia de los proveedores en la cadena productiva

\begin{tabular}{|c|c|c|c|c|c|c|c|c|}
\hline \multicolumn{9}{|c|}{ Productor } \\
\hline ๕̆ & $A^{*}$ & B & C & D & E & $\mathbf{F}$ & G & Grado \\
\hline o & UP1 & UP2 & UP3 & UP4 & UP5 & UP6 & UP7 & \\
\hline $\mathrm{p} 1$ & 0 & 0 & 0 & 1 & 0 & 1 & 0 & 2 \\
\hline p2 & 1 & 0 & 1 & 1 & 0 & 0 & 0 & 3 \\
\hline p3 & 1 & 0 & 0 & 0 & 0 & 1 & 0 & 2 \\
\hline p4 & 0 & 1 & 0 & 0 & 0 & 1 & 0 & 2 \\
\hline p5 & 1 & 0 & 0 & 0 & 0 & 0 & 1 & 2 \\
\hline p6 & 1 & 0 & 1 & 1 & 0 & 1 & 0 & 4 \\
\hline p7 & 1 & 1 & 1 & 1 & 1 & 0 & 0 & 4 \\
\hline P8 & 1 & 0 & 1 & 1 & 0 & 0 & 0 & 3 \\
\hline P9 & 1 & 0 & 1 & 0 & 0 & 0 & 1 & 3 \\
\hline p10 & 0 & 1 & 0 & 0 & 0 & 0 & 1 & 2 \\
\hline p11 & 0 & 0 & 0 & 0 & 1 & 0 & 1 & 2 \\
\hline p12 & 1 & 1 & 1 & 1 & 1 & 1 & 0 & 6 \\
\hline p13 & 0 & 0 & 0 & 1 & 1 & 0 & 1 & 3 \\
\hline p14 & 1 & 0 & 0 & 0 & 1 & 0 & 0 & 2 \\
\hline
\end{tabular}




\begin{tabular}{|c|c|c|c|c|c|c|c|c|}
\hline \multicolumn{1}{|c|}{ Productor } \\
\hline \multirow{2}{*}{$\frac{0}{0}$} & A* & B & C & D & E & F & G & Grado \\
\cline { 2 - 9 }$z$ & UP1 & UP2 & UP3 & UP4 & UP5 & UP6 & UP7 & \\
\hline p15 & 1 & 0 & 0 & 0 & 0 & 0 & 0 & 1 \\
p16 & 1 & 0 & 0 & 0 & 0 & 0 & 0 & 1 \\
\hline
\end{tabular}

Fuente: Elaboración propia con base en la recopilación de la información.

* Asval Flowers Co.

A su vez, la topología del conglomerado con respecto a los proveedores identificados en la cadena de valor -representado en la figura 9-, muestra los sentidos de interrelación entre los productores locales y el grado de participación entre estos, ya que entre más lejos se encuentra un nodo de los demás, mayor es la dependencia con el nodo "hub", lo cual se puede interpretar de la siguiente manera: la empresa Asval Flowers Co. (nodo "hub") realiza compras con todos los proveedores identificados en esta red, en donde mayoritariamente son compras compartidas con otros productores locales.

Figura 9. Topología del conglomerado productor con respecto a los proveedores.

*El peso se indica en las líneas de interconexión.

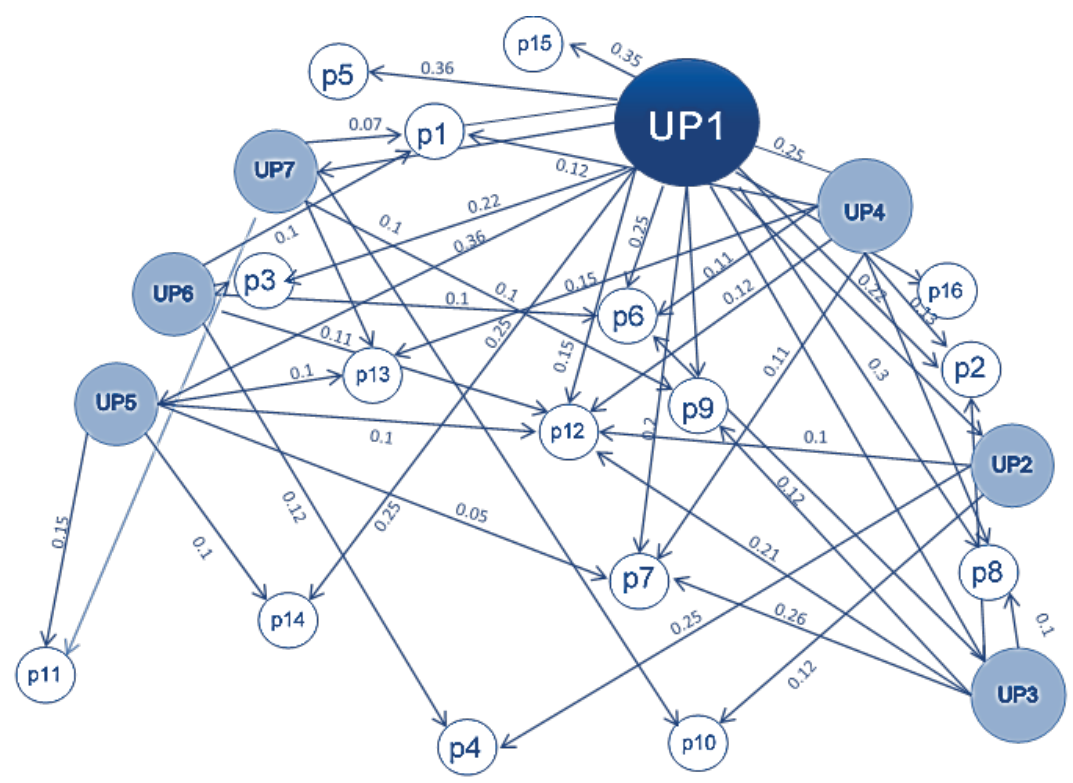

Fuente: Elaboración propia con base en recopilación de información, mediante programa PyCX basado en Python, (https://www.python.org/) 
Lo anterior permite una considerable reducción en sus costos, plazos promedio de pago superiores a los 30 días y una garantía en la proveeduría para todos los integrantes del conglomerado floricultor; a su vez, los demás participantes realizan compras con por lo menos cuatro proveedores, como por ejemplo UP4 con siete de ellos, UP3 con seis, Up5 y Up6 con cinco cada uno; mientras que Up2 y Up7 con cuatro respectivamente.

De igual forma permite identificar el poder de negociación de los proveedores, siendo ( $p 12$ ) el que tiene un mayor peso, debido a que provee a seis de los siete productores locales, seguido de (p6) y (p7) que dan servicio de proveeduría a 4 de estos. Además, se puede identificar que los proveedores ( $p 15)$ y ( $p 16)$ solo tienen relaciones comerciales con Asval Flowers Co. (UP1), esto debido a que son los proveedores ubicados en Holanda y Estados Unidos, respectivamente.

Una vez evaluados los demás componentes del modelo del conglomerado propuesto, es preciso describir la relación entre la eficiencia colectiva y el mejoramiento (upgrading) del desempeño del sector, así como identificar los patrones de gobernanza de la cadena de valor en la que participan. Esto con el fin de proveer información que propicie la mejora del desempeño de las unidades de producción del sector que les permita permanecer competitivas en el mercado global, lo que impacta en el crecimiento económico del estado.

Con base en la opinión de los participantes, la eficiencia colectiva será posible solamente si se mantiene la política de innovación constante en sus procesos, se desarrollan procesos de forma conjunta, la innovación funcional es constante y se comparte equipo entre ellos (Véase Figura 10); lo anterior propicia el incremento de la competitividad de la red y, a su vez, evaluar el desempeño del mismo (upgrading); es decir, la competitividad organizacional resulta de la alineación de las competencias organizacionales con respecto a las oportunidades que se presentan en el entorno; esto mediante la evaluación de su capacidad de mejoramiento del desempeño, la cual se define como innovar para incrementar el valor agregado (Cohelo, 2007: 172).

Figura 10. Eficiencia colectiva.

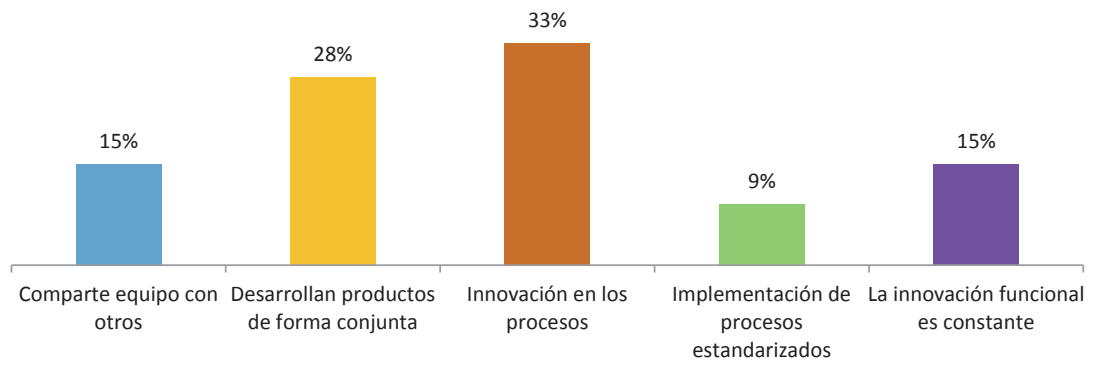

Fuente: Elaboración propia con base en recopilación de información. 
El concepto de mejoramiento (upgrading), de conformidad con Coelho (2007), comenzó a emplearse en el marco de los estudios sobre la competitividad internacional, por lo tanto, el upgrading está íntimamente ligado a la innovación de productos, de procesos y funciones que permiten mejorar el valor agregado de sus grupos de interés, dando paso a la diversificación de productos y oferta de productos complementarios o adicionales como lo son la logística, marketing comercial y distribución.

\section{Conclusiones}

El presente estudio permitió evaluar el grado de conformación del conglomerado florícola del estado de Baja California, Méjico, así como medir su nivel de interrelación productiva y comercial, esto con el fin de que coadyuve a incrementar su competitividad mediante la eficiencia colectiva. Por lo tanto, considerando el objetivo de esta investigación, se concluye que previamente no existía referente del desempeño del conglomerado estudiado, el cual pertenece al sector primario y centra sus actividades en la cadena de valor.

Los hallazgos más representativos demuestran que los integrantes del conglomerado en su mayoría son Mipymes, ubicadas principalmente en los municipios de Playas de Rosarito, Tijuana y Ensenada, las cuales mantienen asociaciones estratégicas por lo menos con un participante del conglomerado, y se identifica a Asval Flowers Corporation como el nodo concentrador que emplea a los demás productores participantes, obteniéndose una densidad representativa en todos los casos, lo que indica que la red posee una amplia interrelación entre sus partes, lo que demuestra que existe una integración horizontal entre los competidores del sector florícola, sus proveedores y clientes mayoristas.

Lo anterior, derivado de la creciente demanda por parte del mercado estadounidense con respecto a los productos florícolas y al número de productores locales que trabajan por periodos intermitentes de tiempo sus tierras, que ofrecen una oportunidad laboral en la que todos trabajan de manera colaborativa bajo las especificaciones de sus clientes comerciales, para lograr la reactivación de la economía local mediante la generación de empleo y el consumo de materias primas, equipo, herramientas y servicios logísticos locales, entre otros. Es decir, con base en lo anterior se presume un crecimiento de participación en el mercado del $50 \%$ para el próximo año.

Las estrategias propuestas por la empresa líder para lograr la sostenibilidad del conglomerado se centran en las innovaciones realizadas en sus procesos productivos, a fin de motivar cada día, que más empresarios 
de Mipyme de este sector participen, ofreciéndoles capacitación y contratación permanente de jornaleros (los cuales rotaban debido la escasez de trabajo permanente), quienes trabajan de forma planificada en los diferentes predios (Unidades económicas), lo cual incrementa la motivación de los trabajadores y disminuye la rotación de personal y. por consiguiente, la constante capacitación.

De igual forma, se pudo constatar que Asval Flowers Co. es una empresa emprendedora que aporta al crecimiento de la floricultura del estado de Baja California, mediante el diseño de nuevas técnicas y la iniciativa de conformar una agrupación de floricultores locales que hasta hace poco tenían sus tierras improductivas, derivado de la falta de capital y una adecuada dirección, aparte de que la mayoría de sus propietarios trabajan bajo la informalidad y carecen de estudios sobre administración de negocios. Para subsanar las deficiencias operativas con las que cuenta el conglomerado, la empresa líder ha diseñado un sistema integral de transporte de cadena en frío que prolonga la vida y calidad de la flor, aprovechando con ello la ventaja para exportar a los distribuidores mayoristas del mercado californiano en los Estados Unidos.

Cabe destacar que, dentro de la interacción mostrada entre los participantes, sobresale la capacidad demostrada por la empresa líder de negociar con proveedores locales e internacionales, los materiales, maquinaria e insumos requeridos por todos los propietarios de predios dedicados a esta actividad, con la finalidad de asegurar la calidad solicitada por los clientes extranjeros, mediante una adecuada administración de costos que, a su vez, les permite homologar los precios.

En la actualidad, el sector florícola advierte como una necesidad, salvaguardar el ambiente, es por ello que los trabajadores del campo han diseñado un Manual de Buenas Prácticas de la floricultura que les permite optimizar el uso de los recursos naturales con los que se cuenta y prevenir la contaminación, mediante el diseño de procesos y procedimientos eficientes, el uso responsable de materiales, el reúso de los desechos (como composta y/o combustible orgánico) y la creación de productos amigables con el ambiente, contribuyendo con esto en una disminución en los impactos ambientales negativos.

La empresa líder ha hecho uso de la técnica de esterilización mediante vapor para la eliminación de las plagas, que proporciona mejores resultados y permite volver a utilizar la tierra prácticamente de inmediato; sin embargo, pese al alto costo del combustible, los costos totales de la implementación de esta técnica alternativa son competitivos frente al uso de bromuro de metilo y con un elevado grado de aceptación por parte 
de los miembros de la cadena de valor, debido a que la desinfección de cantidades limitadas de sustrato convierte a esta opción, desde un punto de vista técnico y económico, en la más eficiente hasta el momento (Valenzuela y Aceves, 2016: 89).

\section{Referencias bibliográficas}

1. ANDERSSON, Thomas, SCHWAAG-SERGER, Silvia, SÖRVIK, Jens, \& WISE, Emily. (2004). The Cluster Policies Whitebook. Estocolmo: International Organization for Knowledge Economy and Enterprise Development (IKED). Disponible en internet http://www.clusterpolisees3.eu/resources/cms/documents/2004_The_Cluster_ Policies_Whitebook.pdf. Consultado el 4.03.2017

2. BERNAL, Blanca, PORTAL, Malena \& DUNIESKY Feitó. (2016). Modelo para evaluar el grado de conformación del conglomerado floricultor de Baja California: un enfoque de cadena de valor. En: Revista internacional Ciencia y Técnica Administrativa $N^{\circ}$ 4. Méjico: Universidad Autónoma de Baja California.

3. BERNAL, Blanca, RAMíREZ, Beatriz \& SEVILLA, Maricela (2014). Programa de mejora del desempeño en modelos de negocio de floricultura. Ponencia en el XVIII Congreso Internacional de Investigación en Ciencias Administrativas Academia de Ciencias Administrativas, A.C. (ACACIA). Memoria digital. Tijuana (Méj): Universidad Autónoma de Baja California.

4. CERDA, Jaime \& VILLAROEL, Luis. (2008). Coeficiente de Kappa. En: Revista Chilena Pediatría No 79. Santiago de Chile: Pontificia Universidad Católica de Chile.

5. COHELO, Alfredo. (2007). Eficiencia colectiva y upgrading en el clúster del tequila. En: Revista Análisis Económico, Vol. XXII, No 49. Méjico D.F.: Universidad Autónoma Metropolitana - Azcapotzalco.

6. GEYER, Robert \& CAIRNEY, Paul. (2015). Handbook on Complexity and public policy. Cheltenham, UK: Edward Elgar.

7. MÉJICO. Instituto Nacional de Estadística y Geografía. (2015). Directorio Estadístico Nacional de Unidades Económicas. Disponible en Internet: http:// www3.inegi.org.mx/sistemas/mapa/denue/default.aspx. Consultado 16.08.2016

8. MÉJICO. Secretaría de Agricultura, Ganadería, Desarrollo Rural, Pesca y Alimentación [SAGARPA], (2012). Proyecto estratégico para la recuperación de la capacidad productiva de suelos de uso agrícola del Valle de Mejicali, Baja California. Disponible en Internet: http://www.sagarpa.gob.mx/Delegaciones/ bajacalifornia/Documents/CONVOCATORIAS/2012/PROYECTO\%20PRECAPS\%20 2012\%20BC\%20\%20SAGARPA-SEFOA\%20(27-07-12).pdf. Consultado 02.12.2016

9. MÉJICO. Secretaría de Agricultura, Ganadería, Desarrollo Rural, Pesca y Alimentación. [SAGARPA] (2015). Programas productivos. Disponible en Internet: 
http://www.sagarpa.gob.mx/agricultura/Programas/proagro/Paginas/default. aspx. Consultado 02.12.2016

10. MÉJICO. Servicio de Información Agroalimentaria y Pesquera ([SIAP], 2013, diciembre 20). Anuario Estadístico de la Producción Agrícola. Elaborado por la SAGARPA. Disponible en internet: http://www.siap.gob.mx/cierre-de-laproduccion-agricola-por-cultivo/.Consultado 18.09.2016

11. MITNIK, Félix. (2011). Desarrollo de cadenas productivas, conglomerados y redes empresariales. Herramientas para el desarrollo territorial. En: Fondo Multilateral de Inversiones. Buenos Aires: Banco Interamericano de Desarrollo.

12. PADILLA, Luz Evelia, PÉREZ, Oscar \& REYES, Eliver. (2012). Evaluación de un conglomerado bajo agricultura protegida en Méjico. En: Revista Contaduría y Administración. Volumen 57 N 3. Ciudad de Méjico: Unidad Académica de Contaduría y Administración, Universidad Autónoma de Zacatecas.

13. PORTER, Michael. (1990). La competitividad internacional de las naciones: Fundamentos teóricos. New York: Free Press.

14. PORTER, Michael. (2005). Estrategia competitiva: técnicas para el análisis de los sectores industriales y de la competitividad. Méjico, 42a reimp. Méjico D. F.: Editorial CECSA.

15. SAYAMA, Hiroki. (2015). Introduction to the modeling and analysis of complex system. Binghamton, Nueva York: State University of New York at Geneseo.

16. SERRET, Daniel. (2011). Estudio de la competitividad de clústeres: el caso del clúster TIC del 22@ (Trabajo de grado). Universidad Politécnica de Catalunya (UPC), Barcelona. Disponible en internet: https://upcommons.upc.edu/pfc/ bitstream/2099.1/11149/1/PFC2.pdf. Consultado el 03.03.2016

17. TRADE map (2017). Estadísticas del comercio para el desarrollo internacional de las empresas, Lista de productos florícolas importados. Disponible en internet: https://www.trademap.org/Country_SelProduct_ TS.aspx?nvpm=3|||||0603|||4|1|1|1|2|1|2|1|1

18. WVALENZUELA, J. y ACEVES, Y. (2016). Manual de Buenas Prácticas en la Floricultura de Asval Flowers Co., Tijuana, B.C. Méjico. Consultado 07.11.2016 
En el altiplano andino, y compartido por Bolivia y Perú, se encuentra el bello y misterioso Lago Titicaca, el lago navegable más alto del mundo, en una prodigiosa armonía paisajística con las hermosas cumbres nevadas de la Cordillera Real. El Titicaca -que significa puma de piedra- también es conocido como el Lago Sagrado de los Incas y es considerado epicentro de las tradiciones andinas. Varias leyendas afirman que el gran Imperio Incaico nació en la Isla del Sol. De las profundas aguas de colores cambiantes por el reflejo del arco iris del cielo, y de la planta de totora que crece en sus riberas, surgieron Manco Cápac y Mama Ocllo, hijos de Inti, el dios $\mathrm{Sol}$, quien les encomendó fundar y civilizar el Imperio Inca. La magia y espiritualidad con las que el Titicaca hechizó a los pueblos, se siente en sus islas Amantiní, Uros o Taquiles, en las cuales aún se preservan costumbres y tradiciones de la época precolombina.

Verónica Hernández O.

Politécnico Colombiano.

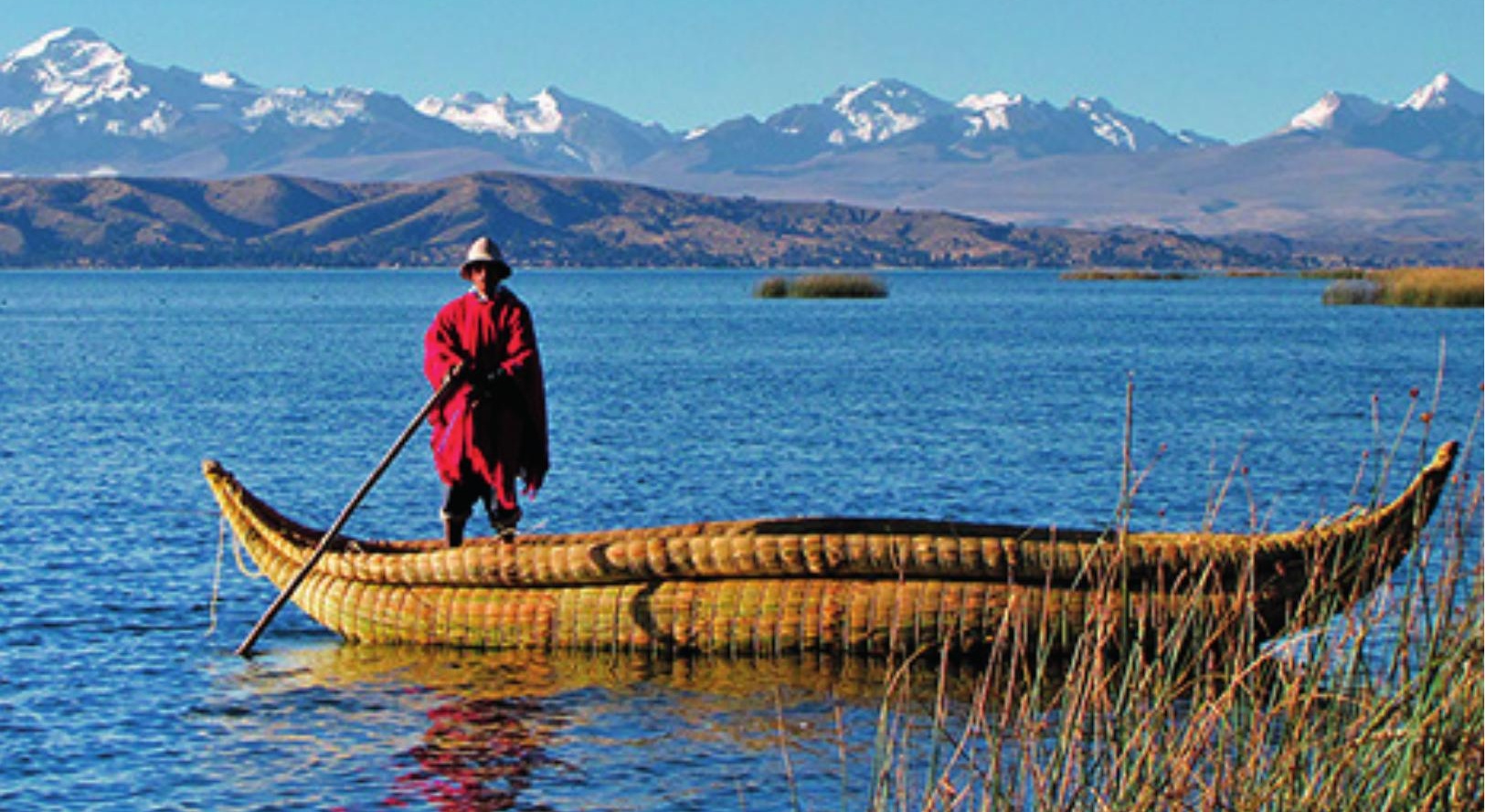

